\title{
Focus on geriatric trauma
}

\section{Pol Maria Rommens ${ }^{1}$ (1)}

Received: 15 February 2019 / Accepted: 26 February 2019

(c) Springer-Verlag GmbH Germany, part of Springer Nature 2019

Life expectancy is high and still growing in our societies. With higher age, the incidence of age-related diseases is increasing. Osteoporosis is a typical disease of the elderly, which is characterized by a decrease of bone mineral density. Therefore, there is a higher risk of fractures due to lowenergy trauma. The aged patient with a fracture is not comparable with an adolescent or adult with a musculoskeletal injury. Aged patients have a restricted physiological reserve and do not afford long and invasive surgeries. In addition, the strength of the fractured bone is lower. Consequently, the prerequisites for fracture care are different. Conservative management plays a more important role. However, surgical treatment is obligatory in patients with unstable fractures of the spine, pelvis, hip and lower extremities. Preoperative time, in which the elderly patients are bedridden and suffer from severe pain, must be as short as possible. Due to less cortical and trabecular bone, fractures have a different morphology and are often complex. Methods of stabilization must be adapted. Timing, invasiveness and type of surgical fixation of geriatric fracture patients must take the specific characteristics of patient and fracture into account. In this focus on geriatric trauma, five authors discuss different aspects of geriatric patients and geriatric fracture care.

In his review, Rommens [1] presents a paradigm shift in the treatment of geriatric fractures. The original principles of operative fracture stabilization were anatomical reduction by open means, stable internal fixation, preserving blood supply and early postoperative motion. Subsequently, anatomical reduction was reserved for articular fractures only, whereas closed, bridging osteosynthesis was introduced for diaphyseal fractures. Biological osteosynthesis was introduced and focused especially on the preservation of blood supply of the bone. In geriatric patients, restoring stability becomes more important than anatomy and biology. Surgery must

Pol Maria Rommens

pol.rommens@unimedizin-mainz.de

1 Department of Orthopaedics and Traumatology, University Medical Center, Johannes Gutenberg-University,

Langenbeckstrasse 1, 55131 Mainz, Germany be minimal invasive; the implants use long bony corridors for bridging or splinting. Conservative treatment is a valid alternative in elderly patients with solitary lesions and low functional demands.

Schmidt et al. [2] performed a logistic regression analysis on 1589 adult patients with isolated mild traumatic brain injury to assess the odds of any in-hospital adverse event by age group, adjusted for gender and comorbidities. An exponential increase of adverse events was observed after the age of 75 years. Most important complications were urinary tract infection, delirium, respiratory complications and early complications in trauma. These results demonstrate that geriatric trauma patients, who need surgical interventions, are at risk for complications during their hospital stay. They need short preoperative times, minimal invasive surgery and early postoperative mobilization to prevent these complications.

Sermon et al. [3] implemented a clinical pathway to enable early surgery after geriatric hip fracture. They compared 744 patients before with 817 patients after implementation of this pathway. Early surgery, which was defined as surgery within the next calendar day, was obtained in $47.6 \%$ versus $85.7 \%$ of patients before and after implementation. There was a significant reduction in length of in-hospital stay, but not of 30 days and 6 months mortality in the group with early surgery. Readmission rate within 90 days was similar in both groups. Data of the rate of in-hospital complications are not available. The results show that mortality of geriatric patients with hip fractures rather is depending on the type, number and severity of comorbidities than on the time of the surgical intervention. A reduction of length of in-hospital stay may nevertheless be beneficial in reducing in-hospital complications. A multidisciplinary approach has proven to be beneficial for the outcome of geriatric patients.

Zajonz et al. [4] identified independent risk factors for early periprosthetic joint infection following hemiarthroplasty in 305 patients with 312 femoral neck fractures. Median age of all patients was 83 years. There were 5.1\% periprosthetic joint infections. Risk factors were high preoperative CRP levels, chronic glucocorticoid use, higher body mass index and prolonged surgery. Length of in-hospital 
stay was doubled and in-hospital mortality 4.6 times higher in patients with an infection. There should be a higher index of suspicion for geriatric patients with these characteristics. Signs of imminent infection must be recognized early and further development of the infection prevented by immediate surgical debridement and rinsing of the wound.

Kim et al. [5] present a series of six geriatric patients with an isolated iliac wing fracture, which were treated with a minimal invasive screw fixation. Fragility fractures of the pelvis are an entity with an increasing frequency. There is an ongoing debate on how to treat these fractures. Minimal invasive surgical techniques have been developed, which use long bone corridors for screw implantation. Iliac wing fractures are a rare but debilitating entity in geriatric pelvic fractures. The very good clinical results in this case series underline the efficacy of this customised surgical technique.

This focus on geriatric trauma is a compilation of a review and four original articles, which highlight different aspects of geriatric trauma care. They underline that the geriatric patient is in many aspects not comparable with the younger, healthy traumatized patient. Elderly patients are at higher risk of general and local complications, which endanger gratifying outcome. The geriatric trauma patient, therefore, needs an adjusted treatment regimen. The role of age for in-hospital adverse events, the importance of conservative treatment or early surgical intervention, the risk factors for early periprosthetic infection, the need for minimal invasive surgery and reduction of length of in-hospital stay are all elements, which need specific attention and are presented in the contributions of this focus on. I wish you enjoyable reading and new insights for the better care of your patients.
Funding None.

\section{Compliance with ethical standards}

Conflict of interest The author declares that he has no conflicts of interest.

\section{References}

1. Rommens PM. Paradigm shift in geriatric fracture care. Eur J Trauma Emerg Surg. 2019. https://doi.org/10.1007/s00068-01901080-x.

2. Schmidt BR, Moos RM, Könu-Leblebicioglu D, Bischoff-Ferrari HA, Simmen HP, Pape HC, Neuhaus V. Higher age is a major driver of in-hospital adverse events independent of comorbid diseases among patients with isolated mild traumatic brain injury. Eur J Trauma Emerg Surg. 2019. https://doi.org/10.1007/s0006 8-018-1029-1.

3. Sermon A, Rochus I, Smeets B, Metsemaekers WJ, Misselyn D, Nijs S, Hoekstra H. The implementation of a clinical pathway enhancing early surgery for geriatric hip fractures: how to maintain a success story? Eur J Trauma Emerg Surg. 2019. https://doi. org/10.1007/s00068-018-1034-4.

4. Zajonz D, Brand A, Lycke C, Özkurtul O, Theopold J, Spiegl UJA, Roth A, Josten C, Fakler JKM. Risk factors for early infection following hemiarthroplasty in elderly patients with a femoral neck fracture. Eur J Trauma emerg Surg. 2019. https://doi. org/10.1007/s00068-018-0909-8.

5. Kim WY, Lee SW, Kim KW, Kwon SY, Choi YH. Minimally invasive surgical treatment using 'iliac pillar' screw for isolated iliac wing fractures in geriatric patients: a new challenge. Eur J Trauma Emerg Surg. 2019. https://doi.org/10.1007/s00068-018-1046-0. 